group $(102 / 176)(p<0.01)$. Type $B$ and $C$ lesions in older group $(180 / 208)$ were significantly higher than in the young group (82/ 176) $(p<0.01)$, while type A lesions $(28 / 208)$ were significantly lower than in the young group $(94 / 176)(p<0.01)$. Multivessel lesions and diffuse calcified lesions in elderly patients with coronary artery disease were significantly higher than in young patients. Conclusions Multivessel lesions and diffuse calcified lesions in elderly patients with coronary artery disease are significantly higher than in young patients, suggesting that elderly patients with coronary artery disease are usually in severe condition and poor prognosis.

\title{
GW23-e0960 CLINICAL CHARACTERISTICS OF 208 CASES OF CORONARY ANGIOGRAPHY IN ELDERLY PATIENTS WITH CORONARY ARTERY DISEASE
}

doi:10.1136/heartjnl-2012-302920n.20

Wei Xiao-jun, Liao Wei, Xie Dong-yang, Wei Xiao-jun. Department of Cardiology, the first affiliated Hospital of Gannan Medical College, GanZhou, 341000, China

Objectives To explore clinical features of coronary angiography (CAG) in elderly patients with coronary artery disease.

Methods Selective CAG was performed by Judkins method. Coronary heart disease was defined as at least a subepicardial vascular diameter stenosis greater than or equal to $50 \%$ (visual method). Coronary arteries were divided into three main branches, namely left anterior descending artery, left circumflex artery and right coronary and the left main coronary artery lesion was treated as two lesions. Lesion sub-type was divided into A, B and C. Type A lesions were less than or equal to $10 \mathrm{~mm}$ in length, with localised lesions and no calcification; type B lesions were $10-20 \mathrm{~mm}$ in length and moderate calcification; type $\mathrm{C}$ lesions were greater than or equal to $20 \mathrm{~mm}$ in lesion, diffuse stenosis or severe calcification. Results Multi-vessel lesions of the older group (149/208) were significantly higher than in the young group (74/176), while single vessel lesions (59/208) were significantly lower than in the young 